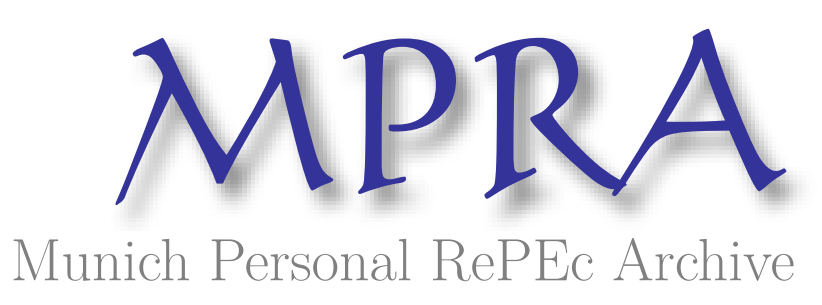

\title{
On Hidden Problems of Option Pricing
}

Olkhov, Victor

TVEL

August 2016

Online at https://mpra.ub.uni-muenchen.de/87173/

MPRA Paper No. 87173, posted 07 Jun 2018 10:08 UTC 


\title{
On Hidden Problems of Option Pricing
}

\author{
Victor Olkhov \\ TVEL, Moscow, Russia \\ victor.olkhov@gmail.com
}

\begin{abstract}
This paper gives new look on option pricing and Black-Scholes-Merton equation within economic space point of view. We argue reasons for economic space definition and it's application for options pricing. Our approach allows review classical Black-Sholes-Merton model and discovers hidden complexity of option pricing. We derive Black-Sholes-Merton equation on $n$-dimensional economic space and argue new tough problems.
\end{abstract}

Keywords: Option Pricing, Black-Scholes-Merton Equations, Economic Space

JEL: C500, C520, C530, C600, G110, G130 


\section{Introduction.}

This paper presents new approach to classical options pricing model that is based on most famous economic equations derived by Black, Scholes and Merton (BSM) more then forty years ago (Black and Scholes [1973]; Merton [1973]). We study options on stock price of corporations, banks or any economic agents. Our approach utilizes economic space that permits describe economic variables of economic agents and their Value in particular as function of time and coordinates on economic space. Option price also becomes a function of coordinates on economic space. Derivation the BSM equation on economic space opens hidden problems for assets and options price modeling and presents many questions. Approach based on economic space notion has nothing common with spatial economics (Perroux [1950]; Fujita [2012]).

Current economic and financial models as well as assets and options price models deal with economic variables like Demand and Supply, Profits and Debts, Corporate Value and Liabilities treating them as function of time. Financial and economic models establish relations between these functions of time and describe dependence of one economic variable upon the others. We propose that extreme complexity of economics and finance requires more improved technique then relations between functions of time. To develop economic modeling capacity let's move from modeling economic variables as functions of time to treating them as functions of time and coordinates. That extension requires introduction of certain economic space where economic and financial variables can be described as functions of time and coordinates. Such economic space (Olkhov, 2016a; 2016b) was introduced as generalization of common risk ratings of economic agents that regularly published by international rating agencies like Fitch (Dyke and Jenning [2015]), S\&P (Kraemer and Vazza [2012]), Moddy's (Metz and Cantor [2007]) etc. Current risks ratings practice can be extended and adopted as basis for economic space. Risk ratings of economic agents can play role of their coordinates on economic space. Such treatment allows study evolution of economic agents on economic space and model behavior of economic variables as functions of coordinates and time. That approach allows review assumptions and derivation of BSM equation on economic space.

The rest of the paper is organized as follows. In the next section we argue reasons for economic space definitions and explain possible advantages for economic and financial modeling and option pricing. Then we discuss option pricing BSM model on economic space 
and outlines additional hidden problems of assets pricing and derivatives theory. Main results are derived in Appendix.

\section{Economic Space.}

Economic space (Olkhov, 2016a; 2016b) was introduced with goal to establish basis for mapping position of each economic agent of entire economics. Dynamics of separate economic agents can be described as evolution of their coordinates on economic space. Up now economic variables like Assets and Liabilities, Value and Capital, Demand and Supply are treated as function of time. Introduction of economic space permits describe economic and financial variables of each economic agent as functions of time and coordinates. That allows model changes of economic variables of particular economic agent like Assets and Liabilities, Value and Debts etc., by methods and technique of mathematical physics equations and definitely enlarges capacity of economic models. Here we present brief reasons for economic space definition.

Definition of economic space utilizes treatment of economic agents as primary elements of entire economics and finance but is completely different from agent-based modeling (Tesfatsion and Judd, 2005). Main idea is clear and simple: economic agents as corporations and banks, householders and investors, etc., are described by set of economic variables as Demand and Supply, Consumption and Savings, Investments and Value and etc. Economic variables of economic agents constitute variables of entire economics and finance. Correct choice of "independent" economic agents permits aggregate their extensive economic and financial variables and determine Demand and Supply, Assets and Credits of entire economics. Let's call economic agents "independent" if sum of their extensive economic variables like Value and Profits, Assets and Liabilities and etc. equal corresponding variables of entire economics. Up now economic variables of economic agents and variables of economics and finance are treated as functions of time. Relations between functions of time describe dependences of Demand and Supply, GDP and Investments, etc., on other economic and financial variables. Such scheme hardly restricts capacity of economic modeling. Definition of economic and financial variables as functions of time and coordinates on certain space permits usage of mathematical physics equations and boost economic modeling capability. Introduction of economic space solve that problem and establish description of economic and financial variables of entire economics and finance as well as economic agents as functions of time and coordinates. 
Introduction of economic space allows distribute economic agents of entire economics by their coordinates on economic space. To do so we propose following:

1. Let's assume that international rating agencies can provide risk ratings for all economic agents of entire economics. If so, economic agents can be distributed over finite number of risk grades by their risk ratings. Risk ratings of economic agents can be treated as their coordinates of finite discreet space.

2. Let's assume that generalization of risk ratings methodology allows plot risk ratings on continues space $R^{l}$. If so, risk ratings of economic agents can be treated as their coordinates on $R^{l}$.

3. Let's assume that simultaneous measurements of ratings of $n$ different risks allows distribute economic agents on $n$-dimensional space that can be discreet or $R^{n}$.

Let's define economic space as any mathematical space that is used to map economic agents by their risk ratings as space coordinates. Dimension of economic space is determined by number of different risks for which risk ratings are measured simultaneously. Usage of economic space $R^{n}$ allows distribute all economic agents of entire economics by their coordinates on $R^{n}$. Economic agents are characterized by economic variables like Demand and Supply, Assets and liabilities, Production Function and Capital. Correct aggregates of economic variables of all economic agents determine variables of entire economics. Sum of extensive economic variables of $k$ "independent" agents equals economic variable of the whole group. For example, sum of Value of economic agents equal their total Value. There are certain parallels between description of economic agents with coordinates on economic space and description of physical particles with coordinates on space-time and for brevity we propose to call economic agents as economic particles or e-particles.

Introduction of economic space gives opportunity to use mathematical physics equations and increase capacity of economic and financial modeling. On the other hand it arises many difficult problems and discovers hidden problems of economic modeling. Definition of economic space $R^{n}$ requires extension of methodology of risk measure estimation. Description on economic space $R^{n}$ requires definition of $n$ major risks that have major effects on economic interactions, macroeconomic and financial processes. To determine a reasonable economic space one should estimate current risks and select two, three, four most important risks as main factors affecting contemporary macroeconomics and finance. That permits establish economic space with two or three dimensions and derive appropriate initial distributions of economic variables as functions of most powerful risks. To select most valuable risks one should establish procedures that allow compare influence of 
different risks on economic agents, their economic interactions and processes. Selection of $n$ major risks defines initial representation of economic space $R^{n}$.

It is well known that risks can suddenly arise and then vanish. To describe economic evolution in a time term $T$ it is necessary forecast $m$ main risks that will play major role in a particular time term and define economic space $R^{m}$. This set of $m$ risks defines target state of economic space $R^{m}$. Changes of major risks require modeling transition from initial set of $n$ risk to target $m$ risk. Such transition should describe how initial set of $n$ risks decline its action on economic agents and their economic variables and how the influence of new $m$ risks grows up. Transition from initial set of $n$ main risk to target set of $m$ risks describes the evolution of initial representation $R^{n}$ of to the target one $R^{m}$.

This is only a part of problems that should be solved to establish reasonable description of economic interactions on economic space. Selection of main risks simplifies description and allows neglect "small risks". Risks benchmarking defines a separate tough problem. Risks selection processes can become a part of validation procedure. Selections of major risks give opportunity to validate initial and target sets of risks and to prove or disprove initial model assumptions. It makes possible to compare theory predictions with observed economic data and outlines causes of disagreements.

Introduction of economic space discovers hidden complexity of economics and finance. We propose that risks should be treated as drivers of economic and finance dynamics and absence of any risks cause degradation of development. To demonstrate advantages of economic space approach we present treatment of option pricing and BSM equations. Further for brevity we mention economic space as e-space and study option pricing on e-space $R^{n}$.

\section{The BSM equations on Economic Space}

Definition of e-space allows look on the BSM equations from a new point of view. Let's study options on stock price of companies, corporations, banks, etc. that are mentioned above as economic agents or e-particles. As we stated before risk rates of e-particles play role of their coordinates on e-space. Risk ratings of e-particles are determined by their economic and financial variables. Economic and financial processes can change variables of e-particles and thus change their risk ratings and their e-space coordinates. In other words motion of eparticles on e-space induce changes of their economic and financial variables. For convenience let's assume that positive direction along each axis of e-space points to risk growth and negative direction points to risk decline. Motion of selected e-particle on e-space induce corresponding changes of it's economic variables. For example, Value of e-particle 
can grow up with motion in high-risk area along risk axis $X$ in positive direction as it may follow with growth of high-risk profits. Further, growth of $X$ coordinate can bring this eparticle to unacceptable risks and that can cause fall of this e-particle Value. Thus value of eparticle, as well as other economic and financial variables are determined as functions of time and coordinates of e-particle on e-space. Movements of e-particle on e-space induce corresponding changes of economic variables. These changes can have regular and random components. Now let's study the BSM equation on option price $V$.

The BSM equation that is one of the most recognized equations in financial theory and for price $V$ of option on underlying asset with price $a$ has form:

$$
\frac{\partial V}{\partial t}+r a \frac{\partial V}{\partial a}+\frac{1}{2} \sigma^{2} a^{2} \frac{\partial^{2}}{\partial a^{2}} V=r V
$$

Here $r$ is risk-free interest rate. A simple way to derive the BSM equation (Hull, 2009) is based on assumption that assets price $a$ obeys Brownian motion $d W(t)$ and

$$
\begin{aligned}
& d a=a c d t+a \sigma d W(t) \\
& <d W(t)>=0 ; \quad<d W(t) d W(t)>=d t
\end{aligned}
$$

$c$ - is instantaneous rate of return on security, and $\sigma^{2}-$ is instantaneous variance rate. Option price $V=V(t, a)$ is function of time $t$ and assets price $a$. Operator $<\ldots>$ denotes averaging procedure.

Let's study the BSM equation on $n$-dimensional e-space $R^{n}$. Thus we assume that economics and selected e-particle are under the action of $n$ major risks. Let's threat options on stock price of e-particles. Stock price of selected e-particle is determined by Value of eparticle. Coordinates of e-particle are determined by their risk ratings. Motion of selected eparticle on e-space $R^{n}$ cause changes of its economic and financial variables and changes of it's Value in particular. Stock price is determined by Value of e-particle and it's changes due to motion on e-space induce corresponding changes of stock price. Let's assume that stock price of selected e-particle is determined by random changes $d W$ in time due to (2) and by motion $d \boldsymbol{x}$ on e-space $R^{n}$

$$
d a=a c d t+a \sigma d W(t)+a \boldsymbol{k} \cdot d \boldsymbol{x}
$$

Let's assume that vector $\boldsymbol{k}$ describes the input of e-space coordinates variations $d \boldsymbol{x}$ on stocks price $a$ and $\boldsymbol{k} \cdot d \boldsymbol{x}$ denotes scalar product. Motion of e-particle has regular and random components. Let's assume that motion of e-particle on e-space $R^{n}$ is determined regular speed $\boldsymbol{v}$ and by Brownian walk $d \mathbf{Z}(t)=\left(d Z_{1}, \ldots d Z_{n}\right)$ on e-space $R^{n}$

$$
d \boldsymbol{x}=\boldsymbol{v} d t+d \boldsymbol{Z}(t)
$$


Value of e-particle on $n$-dimensional e-space $R^{n}$ determines it's stock price $a=a(t, \boldsymbol{x})$. Hence option price $V$ on stock price $a=a(t, \boldsymbol{x})$ of selected e-particle cause that option $V=V(t, \boldsymbol{x}, a)$ becomes a function of coordinates $\boldsymbol{x}$ on $n$-dimensional e-space $R^{n}$. Let's assume that random changes $d W$ of stock price in time and vector components of random walks $d \mathbf{Z}(t)=\left(d Z_{1}, \ldots d Z_{n}\right)$ are correlated and their correlations can be presented as follows:

$$
\begin{aligned}
& <d W(t) d Z_{i}(t)>=b_{i} \\
& <d Z_{i}(t)>=0 ;<d Z_{i}(t) d Z_{j}(t)>=\eta_{i j} d t
\end{aligned}
$$

Relations (6) reflect possible correlations between random changes of stock price in time and random walks along risk-axes of e-space $R^{n}$. Relations (7) describe possible correlations of random walks along different risk axes on e-space. As we show, these factors define behavior of option price $V(t, \boldsymbol{x}, a)$. To derive the BSM equation on e-space $R^{n}$ let's follow usual scheme (Hull, 2009). Assumptions (4-7) allow derive (see Appendix) the BSM equation on option price $V=V(t, x, a)$ on $n$-dimensional e-space $R^{n}$ as follows:

$$
\begin{aligned}
& \frac{\partial V}{\partial t}+r a \frac{\partial V}{\partial a}+r x_{i} \frac{\partial V}{\partial x_{i}}+\frac{1}{2} a^{2} q^{2} \frac{\partial^{2} V}{\partial a^{2}}+\frac{a}{2}\left(\sigma b_{i}+k_{j} \eta_{j i}\right) \frac{\partial^{2} V}{\partial a \partial x_{i}}+\frac{\eta_{i j}}{2} \frac{\partial^{2} V}{\partial x_{i} \partial x_{j}}=r V \\
& q^{2}=\left(\sigma^{2}+k_{i} k_{j} \eta_{i j}+2 \sigma k_{i} b_{i}\right) ; i, j=1, \ldots n
\end{aligned}
$$

Equation (8) has additional parameters $k_{i}, b_{i}, \eta_{i j} ; i, j=1, \ldots n$, and all these factors affect behavior of option price $V(t, x, a)$ on e-space $R^{n}$. Sum is taken by all repeated indexes. Equation (8) has $n$ additional variables $\boldsymbol{x}=\left(x_{1}, \ldots x_{n}\right)$ and more complex then (1) and but conform it's diffusive-type equation.

The main diversity between classical BSM equation (1) and equation (8) concern modeling on e-space $R^{n}$. As we discussed above definition of e-space $R^{n}$ requires selection of $n$ major risks that determine behavior of economics and selected e-particle in particular. This set of risks can vary during time to expiration and new risks may become cause major influence on financial and economic dynamics. To forecast e-particle stock price $a(t, \boldsymbol{x})$ and option price $V(t, \boldsymbol{x}, a)$ dynamics one requires foresee set of $m$ major risks that can replace initial set of $n$ risks. Initial set of $n$ major risks that determine initial e-space $R^{n}$ can dissipate and can be replaced by new set of $m$ risks that will determine new e-space $R^{m}$. To develop appropriate description of stock price and option price dynamics during the transition from espace $R^{n}$ to e-space $R^{m}$ one should describe dissipation of $n$ initial risks and model growth of new $m$ risks. This transition dynamics affect behavior of selected e-particle on initial e-space $R^{n}$. Such influence can cause changes of regular and random behavior of stock prices of eparticle as well as changes of random walks of e-particle on e-space $R^{n}$. Hence options pricing should depend on transition model from initial e-space $R^{n}$ to final e-space $R^{m}$. 
Assumptions (2,3) and (4-7) that found the BSM equation (1) or it's generalization on $n$ dimensional e-space $R^{n}$ can describe option pricing model for constant set of major risks only. Any random changes of major risks, decline of some risks and growth of new risks completely change initial model. Moreover, initial assumptions on Brownian property of random behavior of stock price $a$ and random walks of e-particle coordinates $d \boldsymbol{x}$ during transition from initial set of $n$ major risks on e-space $R^{n}$ to final set of $m$ major risks on espace $R^{m}$ might fail. These random properties may depend on transition process and may be different from Brownian motion.

Development of option pricing model on e-space via the BSM-like equations for the case with variable set of major risks requires additional studies and considerations.

\section{Conclusions}

Assets and options price description is a part of economic and financial models. Introduction of economic space gives general approach to economics and finance modeling and allows describe financial variables as functions of time and coordinates. That simple transition allows boost methods and models for economic and financial modeling. Economic space gives way for wide usage of mathematical physics equations for economic modeling and that may increase capacity and adequacy of description. Economic space approach allows treat option pricing from different point of view and derives BSM-like equations (8) on $n$ dimensional economic space $R^{n}$. These equations depend on coordinates of economic space and on additional parameters that describe correlations of different Brownian walks and other factors.

We assume that main contribution of economic space approach to option pricing concern observation that unpredictable risks behavior may cause important changes of the model description. Possible variability of set of major risks that determine initial representation of economic space should disturb the BSM equations and it's solution. Development of consistent option pricing theory on economic space for cases with variable initial and final set of major risks requires additional research.

Introduction of economic space arises many new difficult problems. Option pricing modeling on economic space requires appropriate econometrics and statistics. Up now econometric data sufficient for modeling on economic space are absent. Adequacy of our approach to financial modeling can be proved by comparison of theory predictions with econometric observations. To do that it is required to launch suitable econometric procedures and obtain distributions of economic agents on economic space. It is necessary develop 
procedures that compare influence of different risks on finance, economics and economic agents dynamics and establish suitable procedures for selection of most valuable risks. That requires cooperation of Central Banks and Financial Regulators, Rating Agencies and Market Authorities, Businesses and Government Statistical Bureaus, Academic and Business Researchers, etc. It is necessary to develop unified risk ratings methodologies that can map risk ratings of economic agents on $R^{n}$ and solve many other problems. Hope it might be useful.

\section{Acknowledgements}

I am very thankful to my spouse Irina for her patience while my studies. This work was performed on my own account only without any support from any other source. 


\section{Appendix. Derivation of BSM Equation on Economic Space $\boldsymbol{R}^{\boldsymbol{n}}$}

Option price $V(t, \boldsymbol{x}, a)$ depends on time $t$ to expiration, e-space $R^{n}$ coordinates $\boldsymbol{x}$ and Price $a=a(t, \boldsymbol{x})$ of underlying stocks of e-particle. Let's assume that stocks price $a$ of e-particle follows regular move and Brownian walks $d W(t)$

$$
\begin{aligned}
& d a=a c d t+a \sigma d W(t)+a \boldsymbol{k} \cdot d \boldsymbol{x} \\
& <d W(t)>=0 \quad ;<d W(t) d W(t)>=d t
\end{aligned}
$$

$c-$ is instantaneous rate of return on security, and $\sigma^{2}-$ is instantaneous variance rate. Operator $\langle\ldots>$ denotes averaging procedure. Vector $\boldsymbol{k}$ describes action of e-space coordinates variation $d \boldsymbol{x}$ on change of e-particle stocks price. $\boldsymbol{k} \cdot d \boldsymbol{x}$ is a scalar product. Let's assume that coordinates $\boldsymbol{x}$ of e-particle that defines underlying stocks price also follows regular move and Brownian walk $d \mathbf{Z}(t)$ on the $n$-dimensional e-space $R^{n}$ :

$$
d \boldsymbol{x}=\boldsymbol{v} d t+d \boldsymbol{Z}(t)
$$

Vector $\boldsymbol{v}$ defines regular velocity of e-particle on e-space. Let's assume that components of Brownian processes $d Z_{i}(t)$ along different axes of e-space $R^{n}$ are correlated and follow relations:

$$
<d Z_{i}(t)>=0 ;<d Z_{i}(t) d Z_{j}(t)>=\eta_{i j} d t
$$

As well let's assume that processes $d W(t)$ and $d Z_{i}(t)$ also correlated:

$$
<d W(t) d Z_{i}(t)>=b_{i} d t
$$

These assumptions lead to equation on option price $V(t, x, a)$ :

$$
\begin{aligned}
d V=\frac{\partial V}{\partial t} d t+ & \frac{\partial V}{\partial a} d a+\frac{\partial V}{\partial x_{i}} d x_{i}+\frac{1}{2} \frac{\partial^{2} V}{\partial t^{2}} d t^{2}+\frac{1}{2} \frac{\partial^{2} V}{\partial a^{2}} d a^{2}+\frac{1}{2} \frac{\partial^{2} V}{\partial x_{i}^{2}} d x_{i}^{2}+\frac{1}{2} \frac{\partial^{2} V}{\partial t \partial a} d t d a \\
& +\frac{1}{2} \frac{\partial^{2} V}{\partial t \partial x_{i}} d t d x_{i}+\frac{1}{2} \frac{\partial^{2} V}{\partial a \partial x_{i}} d a d x_{i}+\frac{1}{2} \frac{\partial^{2} V}{\partial x_{i} \partial x_{j}} d x_{i} d x_{j} ; \quad i>j=1, \ldots n
\end{aligned}
$$

The sum is taken over repeated indexes. Taking account of (A.1-A.5) receive:

$$
\begin{aligned}
d V=\frac{\partial V}{\partial t} d t+ & \frac{\partial V}{\partial a}(c a d t+\sigma a d W(t)+a k \cdot(v d t+d Z(t)))+\frac{\partial V}{\partial x_{i}}\left(v_{i} d t+d Z_{i}(t)\right) \\
& +\frac{1}{2} \frac{\partial^{2} V}{\partial t^{2}} d t^{2}+\frac{1}{2} \frac{\partial^{2} V}{\partial a^{2}}(c a d t+\sigma a d W(t)+a k \cdot(v d t+d Z(t)))^{2} \\
& +\frac{1}{2} \frac{\partial^{2} V}{\partial x_{i}^{2}}\left(v_{i} d t+d Z_{i}(t)\right)^{2}+\frac{1}{2} \frac{\partial^{2} V}{\partial t \partial a} d t(c a d t+\sigma a d W(t)+a k \cdot(v d t \\
& +d Z(t)))+\frac{1}{2} \frac{\partial^{2} V}{\partial t \partial x_{i}} d t\left(v_{i} d t+d Z_{i}(t)\right)+\frac{1}{2} \frac{\partial^{2} V}{\partial a \partial x_{i}}(c a d t+\sigma a d W(t)+a k \\
& \cdot(v d t+d Z(t)))\left(v_{i} d t+d Z_{i}(t)\right)+\frac{1}{2} \frac{\partial^{2} V}{\partial x_{i} \partial x_{j}}\left(v_{i} d t+d Z_{i}(t)\right)\left(v_{j} d t+d Z_{j}(t)\right)
\end{aligned}
$$


With accuracy up to $d t$ obtain:

$$
\begin{aligned}
d V=\left[\frac{\partial V}{\partial t}+(\right. & c a+a k \cdot v) \frac{\partial V}{\partial a}+v_{i} \frac{\partial V}{\partial x_{i}}+\frac{1}{2} a^{2}\left(\sigma^{2}+k_{i} k_{j} \eta_{i j}+2 \sigma k_{i} b_{i}\right) \frac{\partial^{2} V}{\partial a^{2}} \\
+ & \left.\frac{\eta_{i j}}{2} \frac{\partial^{2} V}{\partial x_{i} \partial x_{i j}}+\frac{a}{2}\left(\sigma b_{i}+k_{j} \eta_{j i}\right) \frac{\partial^{2} V}{\partial a \partial x_{i}}\right] d t+\sigma a \frac{\partial V}{\partial a}\left[d W(t)+a k_{i} d Z_{i}(t)\right] \\
+ & \frac{\partial V}{\partial x_{i}} d Z_{i}(t)
\end{aligned}
$$

According to common "portfolio" function

$$
B=V(t, \boldsymbol{x}, a)-a \frac{\partial V}{\partial a}-x_{i} \frac{\partial V}{\partial x_{i}}
$$

and obtain that equation on portfolio $B$ does not depends on Brownian walks

$$
\begin{aligned}
& d B=\left[\frac{\partial V}{\partial t}+\frac{1}{2} a^{2}\left(\sigma^{2}+k_{i} k_{j} \eta_{i j}+2 \sigma k_{i} b_{i}\right) \frac{\partial^{2} V}{\partial a^{2}}+\frac{\eta_{i j}}{2} \frac{\partial^{2} V}{\partial x_{i} \partial x_{j}}\right. \\
& \left.+\frac{a}{2}\left(\sigma b_{i}+k_{j} \eta_{j i}\right) \frac{\partial^{2} V}{\partial a \partial x_{i}}\right] d t
\end{aligned}
$$

Similar to standard derivation of the BSM equation for simplest case let's assume that portfolio $B$ grows as riskless rate $r$ :

$$
d B=r B d t
$$

The BSM equation on $n$-dimensional e-space $R^{n}$ for assumptions (A.1-A.5) takes form:

$$
\begin{gathered}
\frac{\partial V}{\partial t}+r a \frac{\partial V}{\partial a}+r x_{i} \frac{\partial V}{\partial x_{i}}+\frac{1}{2} a^{2} q^{2} \frac{\partial^{2} V}{\partial a^{2}}+\frac{a}{2}\left(\sigma b_{i}+k_{j} \eta_{j i}\right) \frac{\partial^{2} V}{\partial a \partial x_{i}}+\frac{\eta_{i j}}{2} \frac{\partial^{2} V}{\partial x_{i} \partial x_{j}}=r V \\
q^{2}=\left(\sigma^{2}+k_{i} k_{j} \eta_{i j}+2 \sigma k_{i} b_{i}\right) ; \text { sum by } i, j=1, \ldots n
\end{gathered}
$$




\section{References}

Black, F. and M. Scholes, (1973). The Pricing of Options and Corporate Liabilities. The Journal of Political Economy, 81, 637-65.

Dyke, H. and S. Jenning, (2015). Global Structured Finance Rating Criteria. Fitch Ratings, 126.

Fujita, M. (2010). The Evolution Of Spatial Economics: From Thünen To The New Economic Geography. The Japanese Economic Review, 61, 1-32.

Hull, J.C. (2009). Options, Futures and other Derivatives, 7th.ed. Englewood Cliffs, NJ: Prentice-Hall.

Kraemer, N. and D. Vazza. (2012). 2011 Annual U.S. Corporate Default Study And Rating Transitions. $S \& P, 1-96$.

Olkhov, V. (2016a). On Economic space Notion, International Review of Financial Analysis, 47, 372-381

Olkhov, V. (2016b). Finance, Risk and Economic space, ACRN Oxford Journal of Finance and Risk Perspectives, Special Issue of Finance Risk and Accounting Perspectives, 5, 209221.

Merton, R. (1973). Theory of Rational Option Pricing. The Bell Journal of Economic and management Sci, 4, 141-183.

Metz, A., R. (2007). Cantor. Introducing Moody's Credit Transition Model. Moody's Investor Service, 1-26.

Perroux, F. (1950). Economic Space: Theory and Applications. The Quarterly Journal of Economics, 64, 89-104.

Tesfatsion, L., K. Judd., ed. (2005). Handbook of Computational Economics, Vol. 2: AgentBased Computational Economics, Elsevier, North-Holland. 